\title{
One-inclusion Hypergraph Density Revisited
}

\author{
Hans Ulrich Simon and Balázs Szörényi
}

August 12, 2009

\begin{abstract}
In this paper, we show that the density of the one-inclusion hypergraph induced by a family of multi-valued functions is bounded by the pseudo-dimension of this family. (The original proof for this fact, that has been published quite recently, makes use of a wrong claim.) We show furthermore that the well-known graph-dimension is another upper bound on the density (which solves an open problem from [4]).
\end{abstract}

\section{Introduction}

Haussler, Littlestone, and Warmuth [2] investigated prediction-strategies for binary classification problems in a setting where the learner receives $n$ random points $n-1$ of which are labeled correctly, and the challenge is to predict the missing label of the $n$ 'th point with a small probability for making a mistake. In this model, it is assumed that the "true labels" are assigned according to an (unknown) function $f$ taken from a (known) class $\mathcal{F}$ of functions. In [2], a clever prediction strategy is designed that employs a data structure named "one-inclusion graph", and the following is shown:

- The density (i.e. the number of edges over the number of nodes) of the one-inclusion graph divided by $n$ upper bounds the probability for making a mistake.

- The VC-dimension of $\mathcal{F}$ (or even of $\mathcal{F}$ restricted to the $n$ given points) upper-bounds the density of the one-inclusion graph.

Thus, if $d$ denotes the VC-dimension, one arrives at a mistake-bound (i.e. a bound on the probability for making a mistake) of the form $d / n$ when the one-inclusion prediction strategy is applied.

Rubinstein, Bartlett, and Rubinstein [4] extended these results as follows:

- They proved a mistake-bound for the one-inclusion prediction strategy that is strictly smaller than $d / n$ (although asymptotically approaching $d / n$ when $d$ is fixed and $n$ goes to infinity).

- They generalized the one-inclusion prediction strategy to multiclass classification, thereby dealing with one-inclusion hypergraphs, and proved a mistake-bound of the form $d / n$ where $d$ denotes the pseudo-dimension of the (known) class $\mathcal{F}$ of multi-valued functions. 
The proofs of these results are based on a further development of the so-called "shiftingtechnique" by Haussler [1]. Specifically, the proof of the second result makes use of a theorem, Theorem 52 in [4], stating that the pseudo-dimension of $\mathcal{F}$ upper-bounds the density of the corresponding one-inclusion hypergraph. In the proof of this theorem, the shifting-technique is applied and it is claimed that shifting cannot lead to a larger pseudo-dimension. This claim, however, is provably wrong. (For a counterexample, see Section A.) In this paper, we provide an alternative proof for Theorem 52 in [4]. We show furthermore that the well-known graph-dimension of $\mathcal{F}$ is another upper bound on the density (which solves an open problem from [4]).

\section{Definitions, Notations, and Observations}

For sake of brevity, let $[k]=\{0,1, \ldots, k\}$. Throughout this paper, the set $R$ is a discretized hyper-rectangle, i.e., $R$ is of the form $\left[k_{1}\right] \times \cdots \times\left[k_{n}\right]$. Note that $n$ is the dimension and $k_{1}, \ldots, k_{n}$ are the side-lengths of $R$.

In the following definition, "P-dimension" stands for "pseudo-dimension", and G-dimension" stands for "graph-dimension". The other dimensions occurring in the definition are introduced for technical reasons (with the GP-dimension being originally introduced in [3]).

Definition 2.1 (Shattered sets, dimensions) Let $F$ be a subset of $R .^{1}$

1. We say that $I=\left\{i_{1}, \ldots, i_{d}\right\} \subseteq\{1, \ldots, n\}$ is $G$-shattered at levels $t_{1}, \ldots, t_{d}$ if, for every $b \in\{0,1\}^{d}, F$ has a non-empty intersection with the set

$$
R_{b}=\left\{f \in R \mid \forall i \in I:\left(f_{i}=t_{i} \text { if } b_{i}=1\right) \text { and }\left(f_{i} \neq t_{i} \text { if } b_{i}=0\right)\right\} .
$$

The $G$-dimension of $F$, denoted as $G$ - $\operatorname{dim}(F)$, is the largest $d$ such that there exists a set I of size d that is G-shattered by $F$ (at d properly chosen levels).

2. We say that $I=\left\{i_{1}, \ldots, i_{d}\right\} \subseteq\{1, \ldots, n\}$ is $G^{\prime}$-shattered at levels $t_{1}, \ldots, t_{d}$ if, for every $b \in\{0,1\}^{d}, F$ has a non-empty intersection with the set

$$
R_{b}=\left\{f \in R \mid \forall i \in I:\left(f_{i} \neq t_{i} \text { if } b_{i}=1\right) \text { and }\left(f_{i}=t_{i} \text { if } b_{i}=0\right)\right\} .
$$

The $G^{\prime}$-dimension of $F$, denoted as $G$-dim' $(F)$, is the largest d such that there exists a set I of size d that is $G^{\prime}$-shattered by $F$ (at d properly chosen levels).

3. We say that $I=\left\{i_{1}, \ldots, i_{d}\right\} \subseteq\{1, \ldots, n\}$ is $P$-shattered at levels $t_{1}, \ldots, t_{d}$ if, for every $b \in\{0,1\}^{d}, F$ has a non-empty intersection with the set

$$
R_{b}=\left\{f \in R \mid \forall i \in I:\left(f_{i} \geq t_{i} \text { if } b_{i}=1\right) \text { and }\left(f_{i}<t_{i} \text { if } b_{i}=0\right)\right\} .
$$

The P-dimension of $F$, denoted as $P$ - $\operatorname{dim}(F)$, is the largest $d$ such that there exists a set $I$ of size d that is P-shattered by $F$ (at d properly chosen levels).

\footnotetext{
${ }^{1}$ You may think of every element of $F$ as a function table for a function $f \in \mathcal{F}$ restricted to the $n$ random points that are given to the learner. Compare with the introduction.
} 
4. We say that $I=\left\{i_{1}, \ldots, i_{d}\right\} \subseteq\{1, \ldots, n\}$ is GP-shattered at levels $t_{1}, \ldots, t_{d}$ if, for every $b \in\{0,1\}^{d}, F$ has a non-empty intersection with the set

$$
R_{b}=\left\{f \in R \mid \forall i \in I:\left(f_{i}=t_{i} \text { if } b_{i}=1\right) \text { and }\left(f_{i}<t_{i} \text { if } b_{i}=0\right)\right\} .
$$

The GP-dimension of $F$, denoted as GP-dim $(F)$, is the largest d such that there exists a set I of size $d$ that is GP-shattered by $F$ (at d properly chosen levels).

5. We say that $I=\left\{i_{1}, \ldots, i_{d}\right\} \subseteq\{1, \ldots, n\}$ is $G P^{\prime}$-shattered at levels $t_{1}, \ldots, t_{d}$ if, for every $b \in\{0,1\}^{d}, F$ has a non-empty intersection with the set

$$
R_{b}=\left\{f \in R \mid \forall i \in I:\left(f_{i}>t_{i} \text { if } b_{i}=1\right) \text { and }\left(f_{i}=t_{i} \text { if } b_{i}=0\right)\right\} .
$$

The $G P^{\prime}$-dimension of $F$, denoted as $G P$-dim' $(F)$, is the largest $d$ such that there exists a set I of size d that is $G P^{\prime}$-shattered by $F$ (at d properly chosen levels).

The following inequalities are easy to verify:

$$
\begin{aligned}
\max \left\{\mathrm{GP}-\operatorname{dim}(F), \mathrm{GP}-\operatorname{dim}^{\prime}(F)\right\} & \leq \mathrm{P}-\operatorname{dim}(F) \\
\mathrm{GP}-\operatorname{dim}(F) & \leq \mathrm{G}-\operatorname{dim}(F) \\
\mathrm{GP}-\operatorname{dim}^{\prime}(F) & \leq \mathrm{G}-\operatorname{dim}^{\prime}(F)
\end{aligned}
$$

Definition 2.2 (One-inclusion hypergraph and corresponding graph) Let $F$ be a subset of $R$.

1. We define the hypergraph $H_{F}$ induced by $F$ as follows. The set of nodes coincides with $F$. For every $1 \leq j \leq n$, and every choice of coordinates $f_{1}, \ldots, f_{j-1}, f_{j+1}, \ldots, f_{n}$, we have (at most) one hyperedge consisting of all nodes $f \in F$ of the form $f=$ $\left(f_{1}, \ldots, f_{j-1}, h, f_{j+1}, \ldots, f_{n}\right)$ for some $1 \leq h \leq k_{j}$ but we exclude those hyperedges that consist of one node only.

2. The graph $G_{F}$ induced by $F$ is defined similarly. The set of nodes coincides with $F$. An edge in $G_{F}$ is a pair of (non-identical) nodes that are of the form

$$
\left(f_{1}, \ldots, f_{j-1}, h, f_{j+1}, \ldots, f_{n}\right),\left(f_{1}, \ldots, f_{j-1}, \hat{h}, f_{j+1}, \ldots, f_{n}\right), h<\hat{h}
$$

provided that

$$
\left\{\left(f_{1}, \ldots, f_{j-1}, t, f_{j+1}, \ldots, f_{n}\right) \mid h<t<\hat{h}\right\} \cap F=\emptyset
$$

The density of a finite (hyper-)graph is defined as the number of (hyper-)edges divided by the number of nodes. Since the node sets of $H_{F}$ and $G_{F}$ coincide, and every hyperedge in $H_{F}$ is represented by at least one edge in $G_{F}$, the following obviously holds:

$$
\operatorname{density}\left(H_{F}\right) \leq \operatorname{density}\left(G_{F}\right)
$$




\section{Main Results}

The following lemma is the main technical contribution of our paper. The recursion used in the proof is similar to the recursion in the original proof of Sauer's Lemma [5] (although Sauer's Lemma is not at all concerned with density). It is different from the recursion used in the proof from [3] for generalized versions of Sauer's Lemma (but could be used as well for the purpose of proving these generalized versions).

Lemma 3.1 Let $F \subseteq\left[k_{1}\right] \times\left[k_{2}\right] \times \cdots \times\left[k_{n}\right]$, and let $G_{F}$ be the graph induced by $F$. Then, $\operatorname{density}\left(G_{F}\right) \leq G P-\operatorname{dim}^{\prime}(F)$.

Proof Throughout the proof, we assume that $k_{1}, \ldots, k_{n} \geq 1$. Note that this can be always achieved by identifying a hyper-rectangle with some dimensions of side-length 0 with the corresponding lower-dimensional hyper-rectangle without dimensions of side-length 0 . Let $d:=\operatorname{GP}_{-\operatorname{dim}^{\prime}}(F)$. It follows that

$$
s:=k_{1}+\cdots+k_{n} \geq n \geq d .
$$

The proof proceeds by induction over $s \geq d$. If $s=d$, then $F$ coincides with $\{0,1\}^{d}$ so that $H_{F}$ is the $d$-dimensional Boolean cube with $2^{d}$ nodes and $d 2^{d-1}$ edges. In this case,

$$
\operatorname{density}\left(H_{F}\right)=\frac{d \cdot 2^{d-1}}{2^{d}}=\frac{d}{2}<d .
$$

Let us assume now that $s>d$ and remember that $n \geq d$. We associate with $F$ the following two sets:

$$
\begin{aligned}
F^{\prime} & :=\left\{f \in F \mid f_{n} \leq k_{n}-1\right\} \cup\left\{\left(f_{1}, \ldots, f_{n-1}, k_{n}-1\right) \mid\left(f_{1}, \ldots, f_{n-1}, k_{n}\right) \in F\right\} \\
F^{\prime \prime} & :=\left\{\left(f_{1}, \ldots, f_{n-1}\right) \mid\left(f_{1}, \ldots, f_{n-1}, k_{n}-1\right),\left(f_{1}, \ldots, f_{n-1}, k_{n}\right) \in F\right\}
\end{aligned}
$$

Let $G_{F}=(V, E), G_{F^{\prime}}=\left(V^{\prime}, E^{\prime}\right)$ and $G_{F^{\prime \prime}}=\left(V^{\prime \prime}, E^{\prime \prime}\right)$. We claim that, with this notation, the following holds:

$$
\begin{aligned}
\operatorname{GP}-\operatorname{dim}^{\prime}\left(F^{\prime}\right) & \leq d \\
\operatorname{GP}-\operatorname{dim}^{\prime}\left(F^{\prime \prime}\right) & \leq d-1 \\
|V| & =\left|V^{\prime}\right|+\left|V^{\prime \prime}\right| \\
|E| & \leq\left|E^{\prime}\right|+\left|E^{\prime \prime}\right|+\left|V^{\prime \prime}\right|
\end{aligned}
$$

This would accomplish the proof of the lemma because

$$
|E| \leq\left|E^{\prime}\right|+\left|E^{\prime \prime}\right|+\left|V^{\prime \prime}\right| \stackrel{(*)}{\leq} d \cdot\left|V^{\prime}\right|+(d-1) \cdot\left|V^{\prime \prime}\right|+\left|V^{\prime \prime}\right|=d \cdot|V|
$$

where the inequality marked " $(*)$ " follows from (5), (6) and the inductive hypothesis. We still have to prove (5), (6), (7) and (8).

As for (5), assume that $1 \leq i_{1}<\cdots<i_{r} \leq n$ is $\mathrm{GP}^{\prime}$-shattered by $F^{\prime}$ at levels $t_{1}, \ldots, t_{r}$, respectively. Since $F^{\prime}$ has side-length $k_{n}-1$ in dimension $n$, we know that either $i_{r}<n$ or 
$i_{r}=n$ and $t_{r} \leq k_{n}-2$. Note that, according to the definition of $F^{\prime}$, every vector $f \in F^{\prime}$ belongs to $F$ too or has coordinate $k_{n}-1$ in dimension $n$ and will belong to $F$ after lifting-up this coordinate to level $k_{n}$. It follows that $i_{1}, \ldots, i_{r}$ is $\mathrm{GP}^{\prime}$-shattered by $F$ at the same levels $t_{1}, \ldots, t_{r}$ (because, even if $i_{r}=n$, it is immaterial whether the $n$ 'th coordinate of a vector is $k_{n}-1$ or $\left.k_{n}\right)$.

As for (6), assume that $1 \leq i_{1}<\cdots<i_{r} \leq n-1$ is $\mathrm{GP}^{\prime}$-shattered by $F^{\prime \prime}$ at levels $t_{1}, \ldots, t_{r}$, respectively. Then, according to the definition of $F^{\prime \prime}, i_{1}, \ldots, i_{r}, n$ is $\mathrm{GP}^{\prime}$-shattered by $\mathcal{F}$ at levels $t_{1}, \ldots, t_{r}, k_{n}-1$, respectively. Thus, (6) must hold.

(7) clearly holds because

$$
\left(f_{1}, \ldots, f_{n-1}, f_{n}\right) \mapsto \begin{cases}\left(f_{1}, \ldots, f_{n-1}, f_{n}\right) & \text { if } f_{n} \leq k_{n}-1 \\ \left(f_{1}, \ldots, f_{n-1}, k_{n}-1\right) & \text { if } f_{n}=k_{n} \text { and }\left(f_{1}, \ldots, f_{n-1}, k_{n}-1\right) \notin F \\ \left(f_{1}, \ldots, f_{n-1}\right) & \text { if } f_{n}=k_{n} \text { and }\left(f_{1}, \ldots, f_{n-1}, k_{n}-1\right) \in F\end{cases}
$$

is a bijection between $V$ and $V^{\prime} \cup V^{\prime \prime}$.

We finally have to verify (8). To this end, we will apply a counting argument that proceeds "line-wise". Let us first fix coordinates $f_{1}, \ldots, f_{n-1}$ and consider the line segment $L$ between $\left(f_{1}, \ldots, f_{n-1}, 0\right)$ and $\left(f_{1}, \ldots, f_{n-1}, k_{n}\right)$. The number of edges on a line is always one less then the number of nodes. Thus, since by (9)

$$
\begin{aligned}
\left|\left\{\left(f_{1}, \ldots, f_{n-1}, h\right) \in V: 0 \leq h \leq k_{n}\right\}\right|= & \left|\left\{\left(f_{1}, \ldots, f_{n-1}, h\right) \in V^{\prime}: 0 \leq h \leq k_{n}-1\right\}\right| \\
& +\left|\left\{\left(f_{1}, \ldots, f_{n-1}\right)\right\} \cap V^{\prime \prime}\right|
\end{aligned}
$$

it holds that the number of edges in $E$ passing along dimension $n$ equals to the sum of $\left|V^{\prime \prime}\right|$ and the number of edges in $E^{\prime}$ passing along dimension $n$.

Consider now edges $e \in E$ that pass along a dimension $1 \leq j \leq n-1$. By reasons of symmetry, we may assume that $j=1$. Fix coordinates $f_{2}, \ldots, f_{n-1}, h$ and consider the line segment $L_{h}$ between $\left(0, f_{2}, \ldots, f_{n-1}, h\right)$ and $\left(k_{1}, f_{2}, \ldots, f_{n-1}, h\right)$. We proceed by case analysis (see Figures 1 and 2 for an illustration of the second case):

Case 1: $h \leq k_{n}-2$.

Then clearly $\left|E \cap L_{h}\right|=\left|E^{\prime} \cap L_{h}\right|$.

Case 2: $h=k_{n}-1, k_{n}$.

We consider both line segments simultaneously. Furthermore, let $L^{\prime \prime}$ be the line segment between $\left(0, f_{2}, \ldots, f_{n-1}\right)$ and $\left(k_{1}, f_{2}, \ldots, f_{n-1}\right)$. The bijection in $(9)$ shows that

$$
\begin{aligned}
& \left|E \cap\left(L_{k_{n}} \cup L_{k_{n}-1}\right)\right|= \\
& \left\{\begin{array}{ll}
\left|E^{\prime} \cap\left(L_{k_{n}} \cup L_{k_{n}-1}\right)\right|+\left|E^{\prime \prime} \cap L^{\prime \prime}\right|-1 & \text { if } V^{\prime \prime} \cap L^{\prime \prime}=\emptyset \text { and } V \cap L_{k_{n}}, V \cap L_{k_{n}-1} \neq \emptyset \\
\left|E^{\prime} \cap\left(L_{k_{n}} \cup L_{k_{n}-1}\right)\right|+\left|E^{\prime \prime} \cap L^{\prime \prime}\right| & \text { otherwise }
\end{array} .\right.
\end{aligned}
$$

(8) follows from the preceding discussion.

Corollary 3.2 Let $F \subseteq\left[k_{1}\right] \times\left[k_{2}\right] \times \cdots \times\left[k_{n}\right]$, and let $G_{F}$ be the graph induced by $F$. Then, $\operatorname{density}\left(G_{F}\right) \leq G P-\operatorname{dim}(F)$. 
dimension $n$
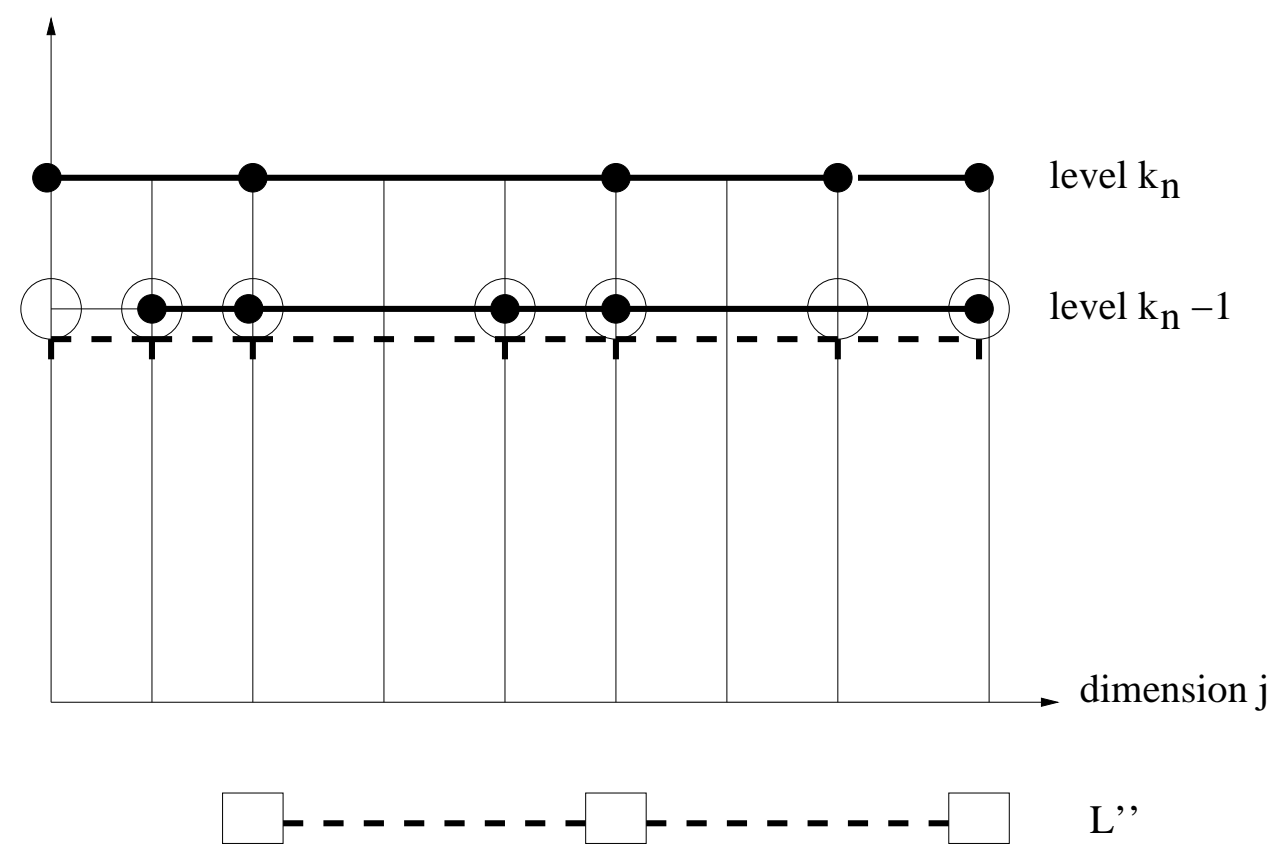

Figure 1: The scenario for edges passing along dimension $j$ at levels $k_{n}$ or $k_{n}-1$, respectively: Solid circles represent nodes from $V$, hollow circles represent nodes from $V^{\prime}$, and squares represent nodes from $V^{\prime \prime}$. Thick solid lines indicate edges from $E$ and thick dotted lines indicate edges from $E^{\prime}$ and $E^{\prime \prime}$. In this example, $\left|E \cap L_{k_{n}}\right|=\left|E \cap L_{k_{n}-1}\right|=4,\left|E^{\prime} \cap L_{k_{n}-1}\right|=6$, and $\left|E^{\prime \prime} \cap L^{\prime \prime}\right|=2$.

dimension $n$

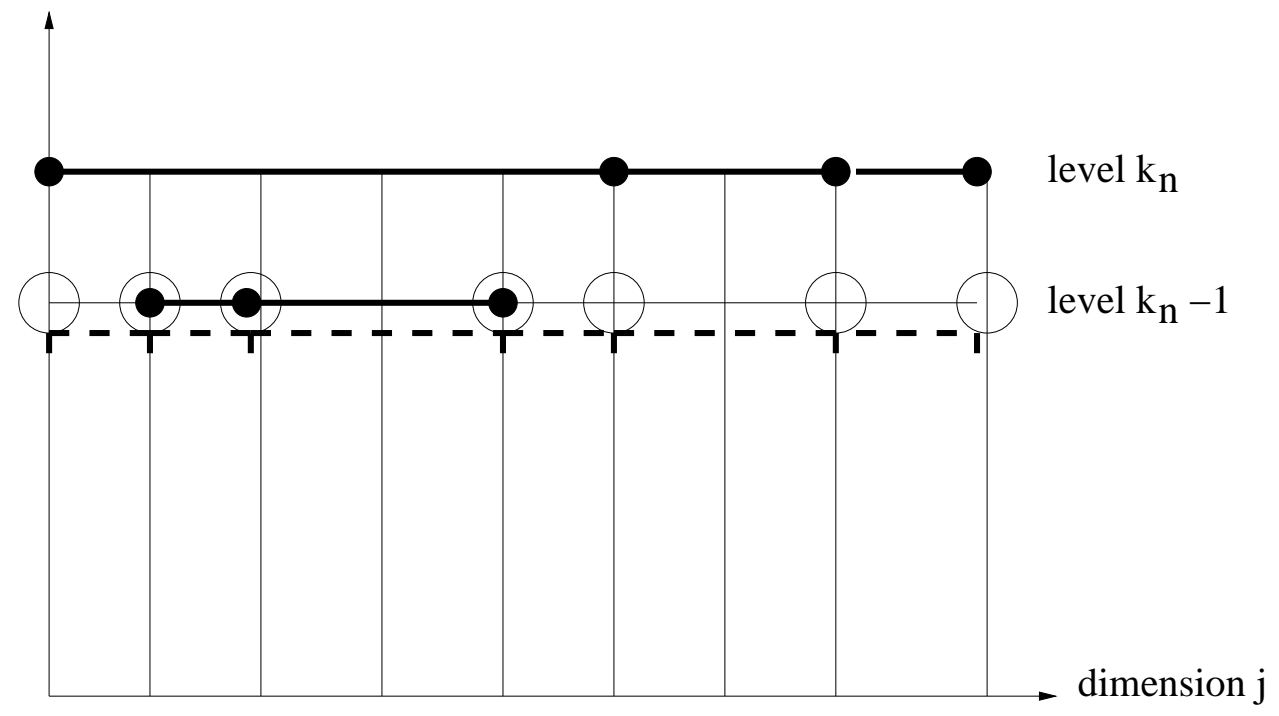

Figure 2: A similar scenario as in Figure 1 but now $V^{\prime \prime} \cap L^{\prime \prime}=\emptyset$. In this example, $\left|E \cap L_{k_{n}}\right|=$ $3,\left|E \cap L_{k_{n}-1}\right|=2,\left|E^{\prime} \cap L_{k_{n}-1}\right|=6$, and $\left|E^{\prime \prime} \cap L^{\prime \prime}\right|=0$. 
Proof The proof is analogous to the proof of Lemma 3.1, but we have to deal with hyperrectangles of the form

$$
R=\left\{l_{1}, \ldots, k_{1}\right\} \times \cdots \times\left\{l_{n}, \ldots, k_{n}\right\},
$$

which makes the notation more cumbersome. Initially, $l_{1}=\cdots=l_{n}=0$, but we are about to apply an inductive argument which leads to higher values of the parameters $l_{i}$. Given $F \subseteq R$ where $R$ is a hyper-rectangle of the form (10), the sets $F^{\prime}$ and $F^{\prime \prime}$ are now defined as follows:

$$
\begin{aligned}
F^{\prime} & :=\left\{f \in F \mid f_{n} \geq l_{n}+1\right\} \cup\left\{\left(f_{1}, \ldots, f_{n-1}, l_{n}+1\right) \mid\left(f_{1}, \ldots, f_{n-1}, l_{n}\right) \in F\right\} \\
F^{\prime \prime} & :=\left\{\left(f_{1}, \ldots, f_{n-1}\right) \mid\left(f_{1}, \ldots, f_{n-1}, l_{n}\right),\left(f_{1}, \ldots, f_{n-1}, l_{n}+1\right) \in F\right\}
\end{aligned}
$$

We may apply a symmetry argument with levels $l_{n}$ and $l_{n}+1$ in dimension $n$ playing the role that levels $k_{n}, k_{n}-1$ were playing before.

Combining Lemma 3.1 and Corollary 3.2 with (1), (2), (3), and (4), we arrive at the following result:

Corollary 3.3 Let $F \subseteq\left[k_{1}\right] \times\left[k_{2}\right] \times \cdots \times\left[k_{n}\right]$, and let $H_{F}$ be the hypergraph induced by $F$. Then,

$$
\begin{aligned}
\operatorname{density}\left(H_{F}\right) \leq \operatorname{density}\left(G_{F}\right) & \leq \min \left\{G P-\operatorname{dim}(F), G P-\operatorname{dim}^{\prime}(F)\right\} \\
& \leq \min \left\{P-\operatorname{dim}(F), G-\operatorname{dim}(F), G-\operatorname{dim}^{\prime}(F)\right\}
\end{aligned}
$$

\section{References}

[1] David Haussler. Sphere packing numbers for subsets of the boolean $n$-cube with bounded Vapnik-Chervonenkis dimension. Journal of Combinatorial Theory, Series A, 69(2):217232, 1995.

[2] David Haussler, Nick Littlestone, and Manfred K. Warmuth. Predicting $\{0,1\}$ functions on randomly drawn points. Information and Computation, 115(2):284-293, 1994.

[3] David Haussler and Phil M. Long. A generalization of Sauer's lemma. Journal of Combinatorial Theory, Series A, 71(2):219-240, 1995.

[4] Benjamin I. P. Rubinstein, Peter L. Bartlett, and J. Hyam Rubinstein. Shifting: Oneinclusion mistake bounds and sample compression. Journal of Computer and System Sciences, 75(1):37-59, 2009.

[5] N. Sauer. On the density of families of sets. Journal of Combinatorial Theory, Series A, 13(1):145-147, 1972. 


\section{A Shifting and Pseudo-dimension}

In [4], the following shifting-operators are used:

- Operator $S_{i, t}$ shifts any vector in $F$ that is located at level $t$ in dimension $i$ to the lowest level in dimension $i$ that is not occupied by another node from $V$ (thereby keeping the remaining coordinates fixed).

- Operator $S_{i}$ is defined as the concatenation $S_{i, k_{i}} \circ \cdots \circ S_{i, 1}$.

It is claimed in [4] that any index set $\mathrm{P}$-shattered by $S_{i}(F)$ is P-shattered by $F$ too. Clearly, this would imply that the $\mathrm{P}$-dimension of $S_{i}(F)$ is upper-bounded by the P-dimension of $F$. The simplest counterexample against this claim is as follows:

$$
\begin{aligned}
F & =\{(0,0),(0,1),(1,1),(1,2)\} \\
S_{2}(F) & =\{(0,0),(0,1),(1,0),(1,1)\}
\end{aligned}
$$

Note that

$$
\mathrm{P}-\operatorname{dim}(F)=1<2=\mathrm{P}-\operatorname{dim}\left(S_{1}(F)\right) .
$$

By induction, we can extend this counterexample so as to get a $d$-dimensional set $F_{d}$ of P-dimension 1 that can be iteratively shifted to a set $\tilde{F}_{d}$ of P-dimension $d$ (which shows that shifting can increase the $\mathrm{P}$-dimension as much as we like):

1. $F_{1}=\{(0),(1)\}$ and $v_{\max }\left(F_{1}\right)=(1)$.

2. $F_{d}=\left(\{0\} \times F_{d-1}\right) \cup\left(\{1\} \times\left(v_{\max }+F_{d-1}\right)\right)$ and $v_{\max }\left(F_{d}\right)=\{1\} \times\left(2 v_{\max }\left(F_{d-1}\right)\right)$.

For example, $F_{2}$ is the simple counterexample that we had discussed first, $v_{\max }\left(F_{2}\right)=(1,2)$, and

$$
\begin{aligned}
F_{3} & =\{(0,0,0),(0,0,1),(0,1,1),(0,1,2),(1,1,2),(1,1,3),(1,2,3),(1,2,4)\}, \\
v_{\text {max }}\left(F_{3}\right) & =(1,2,4) .
\end{aligned}
$$

It easy to show inductively that the following holds:

- The vectors from $F_{d}$ form a chain w.r.t. relation $\leq$ (understood component-wise). This implies that $\mathrm{P}-\operatorname{dim}\left(F_{d}\right)=1$.

- Shifting according to $S_{2} \circ \cdots \circ S_{d}$ transforms $F_{d}$ into $\tilde{F}_{d}=\{0,1\}^{d}$. The latter set has P-dimension $d$. 